\title{
Radiative correction to the lightest neutral Higgs mass in warped supersymmetry
}

\author{
Gautam Bhattacharyya $^{1}$, Swarup Kumar Majee ${ }^{2,3}$, Tirtha Sankar Ray ${ }^{1}$ \\ 1) Saha Institute of Nuclear Physics, 1/AF Bidhan Nagar, Kolkata 700064, India \\ 2) Harish-Chandra Research Institute, Chhatnag Road, Jhunsi, Allahabad 211019, India \\ 3) Department of Physics, University of Calcutta, 92 A.P.C. Road, Kolkata 700009, India
}

\begin{abstract}
We compute radiative correction to the lightest neutral Higgs mass $\left(m_{h}\right)$ induced by the Kaluza-Klein (KK) towers of fermions and sfermions in a minimal supersymmetric scenario embeded in a 5-dimensional warped space. The Higgs is confined to the TeV brane. The KK spectra of matter supermultiplets is tied to the explanation of the fermion mass hierarchy problem. We demonstrate that for a reasonable choice of extra-dimensional parameters, the KK-induced radiative correction can enhance the upper limit on $m_{h}$ by as much as $100 \mathrm{GeV}$ beyond the $4 \mathrm{~d}$ limit of $135 \mathrm{GeV}$.
\end{abstract}

PACS Nos: $12.60 . \mathrm{Jv}, 11.10 . \mathrm{Kk}$

Key Words: Supersymmetry, Warped Extra Dimension, Higgs mass

Introduction: Minimal supersymmetric standard model (MSSM) with superparticles in the $1 \mathrm{TeV}$ range, primarily for its ability to settle the gauge hierarchy problem and for providing a cold dark matter candidate, has emerged as a leading candidate of physics beyond the standard model (SM). A key prediction of MSSM is the existence of a light Higgs $\left(m_{h}<135 \mathrm{GeV}\right)$. If such a light scalar exists, the CERN Large Hadron Collider (LHC) will find it hard to miss. Moreover, if a quantum picture for all interactions including gravity has to be weaved, we have to rely on string theory, which invariably includes supersymmetry (SUSY). Since string theory is fundamentally a higher dimensional theory, a reanalysis of the four-dimensional (4d) MSSM Higgs spectra by embedding the theory in an extra-dimensional set-up is a worthwhile phenomenological exercise.

Randall-Sundrum (RS) type models [1 with a warped space-time geometry, where the bulk is a slice of Anti-deSitter (AdS) space in which the SM/MSSM particles can also penetrate 2, 3, lead to important phenomenological consequences: (i) gauge hierarchy problem is solved thanks to the warp factor, (ii) mass hierarchy of fermions can be explained by their relative localizations in the bulk [4, (iii) the smallness of neutrino masses can be explained [5, (iv) gauge couplings unify if the warped space is supersymmetric [6], (v) SUSY breaking can be realized with a geometrical interpretation [7, (vi) light Kaluza-Klein (KK) gauge boson and fermion states can be captured at the LHC, and some other specific signals, like top flavor-violating decays, can be detected as well [8].

Since Higgs is the most-wanted entity at the LHC, our intention in this paper is to calculate how the upper limit on the lightest supersymmetric neutral Higgs mass changes in the warped extra-dimensional backdrop due to radiative corrections induced by the KK towers of fermions and sfermions. Before we perch on extradimensional details, we mention that even within the $4 \mathrm{~d}$ set-up the Higgs mass receives additional contribution, beyond the MSSM limit of $135 \mathrm{GeV}$, in the next-to-minimal MSSM [9] and in the left-right MSSM [10], to the tune of a few tens of a $\mathrm{GeV}$ in cach case.

5d warped MSSM: The fifth dimension $y$ is compactified on an $S^{1} / Z_{2}$ orbifold of radius $R$. Two 3-branes are located at the orbifold fixed points at $y=(0, \pi R)$. The space-time between the two branes is a slice of $\mathrm{AdS}_{5}$ geometry. The $5 \mathrm{~d}$ metric is given by,

$$
d s^{2}=e^{-2 \sigma} \eta_{\mu \nu} d x^{\mu} d x^{\nu}+d y^{2}, \quad \text { where } \sigma=k|y| .
$$


Above, $1 / k$ is the AdS curvature radius and $\eta_{\mu \nu}=\operatorname{diag}(-1,1,1,1)$. The 3 -brane at $y=0$ is called the Planck brane as the natural mass scale associated with it is $M_{P}$, while the 3-brane at $y=\pi R$ with an effective mass scale $M_{P} e^{-\pi k R}$ can be called a $\mathrm{TeV}$ brane provided $k R \simeq 12$.

We consider a supersymmetric scenario in which not only gravity but all particles can, in principle, access the AdS bulk [3. There are quite a few boons of supersymmetrizing an $\mathrm{AdS}_{5}$ slice, e.g. a possible connection to string theory can be established, new avenues for SUSY breaking can be explored, etc. We concentrate on the structure of matter hypermultiplets and the nature of Yukawa interactions for their special relevance in the computation of radiative corrections to the Higgs mass.

The hypermultiplet $\Phi=\left(\phi^{i}, \Psi\right)$ contains two complex scalars $\phi^{i}(i=1,2)$ and a Dirac fermion $\Psi$. The action can be written as $\left(g \equiv \operatorname{det}\left(g_{M N}\right)\right)[\underline{3}$

$$
S_{5}=-\int d^{4} x \int d y \sqrt{-g}\left[\left|\partial_{M} \phi^{i}\right|^{2}+i \bar{\Psi} \gamma^{M} D_{M} \Psi+m_{\phi^{i}}^{2}\left|\phi^{i}\right|^{2}+i m_{\Psi} \bar{\Psi} \Psi\right] .
$$

Invariance under supersymmetric transformation yields 3 ]

$$
m_{\phi^{1,2}}^{2}=\left(c^{2} \pm c-\frac{15}{4}\right) k^{2}+\left(\frac{3}{2} \mp c\right) \frac{d^{2} \sigma}{d y^{2}}, \text { and } m_{\Psi}=c \frac{d \sigma}{d y},
$$

where $c$ is some arbitrary real number. A generic $5 \mathrm{~d}$ field can be decomposed as 3 ]

$$
\Phi\left(x^{\mu}, y\right)=\frac{1}{\sqrt{2 \pi R}} \sum_{n=0}^{\infty} \Phi^{(n)}\left(x^{\mu}\right) f_{n}(y), \quad \text { where } f_{n}(y)=\frac{e^{s \sigma / 2}}{N_{n}}\left[J_{\alpha}\left(\frac{m_{n}}{k} e^{\sigma}\right)+b_{\alpha}\left(m_{n}\right) Y_{\alpha}\left(\frac{m_{n}}{k} e^{\sigma}\right)\right],
$$

with $s=(4,1,2)$ for $\Phi=\left\{\phi, e^{-2 \sigma} \Psi_{L, R}, A_{\mu}\right\}$. For the detailed formulae of $b_{\alpha}$ and the normalization $N_{n}$ in terms of the Bessel functions $J_{\alpha}$ and $Y_{\alpha}$ (where $\alpha$ is an index for 5 d mass and is a function of $c$ ), see Ref. [3]. Regardless of $Z_{2}$ even or odd nature of KK modes, one obtains an approximate expression of the KK mass:

$$
m^{(n)} \simeq\left(n+\frac{c}{2}-\frac{1}{2}\right) \pi k e^{-\pi k R}, \text { where } n=1,2, . .
$$

Two points are worth noting: (i) Even though $5 \mathrm{~d} N=1$ SUSY is equivalent to $N=2$ in $4 \mathrm{~d}$, the massless sector of the hypermultiplet (from $\phi^{1}$ and $\Psi_{L}$, in our convention) forms an $N=1$ chiral supermultiplet, and (ii) in the conformal limit $(c=1 / 2)$, the massless modes are not localized in the bulk and couple to the two branes with equal strength. For $c<1 / 2$, the zero modes are confined towards the TeV brane, while for $c>1 / 2$, the zero modes are localized closer to the Planck brane.

We now come to the Yukawa interaction. First, we assume that the Higgs boson is localized at the TeV brane, i.e. $H(x, y)=H(x) \delta(y-\pi R)$ [this immediately solves the $\mu$ problem, as $\mu \sim \mathcal{O}(\mathrm{TeV})$ ]. Considering that for each fermion flavor $i$, there are two $5 \mathrm{~d}$ Dirac fermions $\Psi_{i L}(x, y)$ and $\Psi_{i R}(x, y)$, one can write the Yukawa action as $\left(H(x) \equiv H_{u}(x)\right.$ or $\left.H_{d}(x)\right)$

$$
S_{y}=\int d^{4} x \int d y \sqrt{-g} \lambda_{i j(5 d)} H(x)\left(\bar{\Psi}_{i L}(x, y) \Psi_{j R}(x, y)+\text { h.c. }\right) \delta(y-\pi R) .
$$

Recall that each $5 \mathrm{~d}$ fermion field has a bulk mass term, characterized by $c_{i L}$ or $c_{i R}$. For simplicity, we assume that $c_{i} \equiv c_{i L}=c_{i R}$. We now expand the $5 \mathrm{~d}$ fermion fields in zero modes and higher KK modes and obtain the corresponding $4 \mathrm{~d}$ Yukawa couplings. For simplicity, we consider only the diagonal couplings, i.e. ignore quark mixings as their numerical effects are negligible for our calculation. The Yukawa couplings of the zero mode fermions are given by

$$
\lambda_{i}=\lambda_{i(5 d)} k\left(1 / 2-c_{i}\right)\left(1-e^{\left(2 c_{i}-1\right) \pi k R}\right)^{-1} .
$$

Now we assume $\lambda_{i(5 d)} k \sim 1$, and trade the zero mode fermion masses in favor of the corresponding $c_{i}$ (see Table $1)$. This is how the fermion mass hierarchy problem is addressed. We note here that the choice of $c_{i}>1 / 2$ for the first two families helps evade tight constraints $\left(m^{(1)}>\right.$ a few TeV) from FCNC processes [2]. For the third generation, FCNC constraints are not so stringent any way. 


\begin{tabular}{|c|c|c|c|c|c|c|c|c|c|}
\hline$f_{i}$ & $e$ & $\mu$ & $\tau$ & $u$ & $d$ & $c$ & $s$ & $t$ & $b$ \\
\hline$c_{i}$ & 0.61 & 0.52 & 0.40 & 0.62 & 0.57 & 0.52 & 0.52 & -0.50 & 0.26 \\
\hline$m_{i}^{(1)}$ & 1598 & 1508 & 1388 & 1609 & 1562 & 1504 & 1510 & 500 & 1249 \\
\hline
\end{tabular}

Table 1: The $c_{i}$ parameters and $m_{i}^{(1)}$ (in GeV) for different flavors are shown for $k R=11.93$ and $\tan \beta=\left\langle H_{u}^{0}\right\rangle /\left\langle H_{d}^{0}\right\rangle=10$. For this choice, the mass gap between the consecutive $\mathrm{KK}$ states is $m^{(n+1)}-m^{(n)}=1987 \mathrm{GeV}$, irrespective of $c_{i}$. The corresponding $n=1 \mathrm{KK}$ mass for gauge boson is $1490 \mathrm{GeV}$.

We now turn our attention to the Yukawa couplings of KK fermions. We assume KK number conservation at the tree level Higgs coupling with the KK fermions. Inserting the expansion in Eq. (44) in Eq. (6), we derive (again, considering only diagonal couplings):

$$
\lambda_{i}^{(n)}=\lambda_{i(5 d)} \pi m^{(n)} e^{\pi k R}\left[J_{\alpha}\left(\frac{m^{(n)}}{k} e^{\pi k R}\right)+b_{\alpha}\left(m^{(n)}\right) Y_{\alpha}\left(\frac{m^{(n)}}{k} e^{\pi k R}\right)\right]^{2} .
$$

A set of (reasonable) approximations $m_{n} \ll k \sim M_{P}, k R \gg 1$ and $\lambda_{i(5 d)} k \sim 1$ simplifies the above as,

$$
\lambda_{i}^{(n)} \sim \cos ^{2}\left(\left[n-\frac{3}{4} \mp \frac{1}{4}\right] \pi\right),
$$

where $\mp$ correspond to $Z_{2}$ even/odd KK modes. We draw two important conclusions from Eq. (9): (i) all KK Yukawa couplings, regardless of their flavors (i.e. $c_{i}$ values) and KK numbers, are roughly equal being close to unity (more precisely, $\lambda_{i(5 d)} k$ ), and (ii) the KK Yukawa couplings of $Z_{2}$ odd modes are vanishing (since the Higgs is brane-bound).

Radiative correction to the Higgs mass: We recall that in 4d MSSM the lightest neutral Higgs mass at the tree level is lighter than $M_{Z}$, the $Z$-boson mass. More specifically, $m_{h} \leq \min \left(m_{A}, M_{Z}\right)|\cos 2 \beta| \leq$ $\min \left(m_{A}, M_{Z}\right)$, where $M_{A}$ is the pseudo-scalar Higgs mass. The radiative correction to $m_{h}$, dominated by the top quark Yukawa coupling and the masses of the stop squarks, is given by $\Delta m_{h}^{2} \simeq\left(3 m_{t}^{4} / 2 \pi^{2} v^{2}\right) \ln \left(m_{\tilde{t}}^{2} / m_{t}^{2}\right)$. Here, $v^{2}=\left\langle H_{u}^{0}\right\rangle^{2}+\left\langle H_{d}^{0}\right\rangle^{2}=1 /\left(\sqrt{2} G_{F}\right)$ and $m_{\tilde{t}}=\sqrt{m_{\tilde{t}_{1}} m_{\tilde{t}_{2}}}$ is an average stop mass [12].

Here, our goal is to compute $\Delta m_{h}^{2}$ induced by the KK fermions. We shall follow the effective potential technique to implement this correction. We shall ignore the contributions from gauge sector as they are not numerically significant. Dominant effects arise from matter sector. The full one-loop effective potential is given by $V_{1}(Q)=V_{0}(Q)+\Delta V_{1}(Q)$ where, in terms of the field dependent masses $M(H)$, $\Delta V_{1}(Q)=\left(64 \pi^{2}\right)^{-1} \operatorname{Str} M^{4}(H)\left\{\ln \left(M^{2}(H) / Q^{2}\right)-3 / 2\right\}$. The scale dependence of $\Delta V_{1}(Q)$ cancels against that of $V_{0}(Q)$ making $V_{1}(Q)$ scale independent up to higher loop orders. The supertrace is defined through $\operatorname{Str} f\left(m^{2}\right)=\sum_{i}(-1)^{2 J_{i}}\left(2 J_{i}+1\right) f\left(m_{i}^{2}\right)$, where the sum is taken over all members of a supermultiplet. The contribution from the chiral multiplet labeled by the fermion $f$ is given by (with $N_{c}$ as the color factor)

$$
\Delta V_{f}=\frac{N_{c}}{32 \pi^{2}}\left\{m_{\tilde{f}_{1}}^{4}\left(\ln \frac{m_{\tilde{f}_{1}}^{2}}{Q^{2}}-\frac{3}{2}\right)+m_{\tilde{f}_{2}}^{4}\left(\ln \frac{m_{\tilde{f}_{2}}^{2}}{Q^{2}}-\frac{3}{2}\right)-2 m_{f}^{4}\left(\ln \frac{m_{f}^{2}}{Q^{2}}-\frac{3}{2}\right)\right\} .
$$

For illustration, we take only the up-quark chiral multiplet. The field dependent KK quark masses are,

$$
\left(m_{u}^{(n)}\right)^{2}(H)=\left(\lambda_{u}^{(n)}\right)^{2}\left|H_{u}^{0}\right|^{2}+\left(m^{(n)}\right)^{2}
$$

while the KK mass square matrix of the up-squark is obtained as,

$$
M_{\tilde{u}}^{(n) 2}(H)=\left(\begin{array}{cc}
m_{Q}^{2}+\left|H_{u}^{0}\right|^{2}\left(\lambda^{(n)}\right)^{2} & \lambda^{(n)}\left(A_{u} H_{u}^{0}+\mu H_{d}^{0^{*}}\right) \\
\lambda^{(n)}\left(A_{u} H_{u}^{0^{*}}+\mu H_{d}^{0}\right) & m_{U}^{2}+\left|H_{u}^{0}\right|^{2}\left(\lambda^{(n)}\right)^{2}
\end{array}\right)+\left(\begin{array}{cc}
\left(m^{(n)}\right)^{2} & 0 \\
0 & \left(m^{(n)}\right)^{2}
\end{array}\right) .
$$

\footnotetext{
${ }^{1}$ Although, unlike in UED, KK-parity is not automatic in the warped scenario, it is still possible to implement it in a slice of
} $\mathrm{AdS}_{5}$ [11. We assume this parity for simplicity of our analytic computation. This also helps in evading some FCNC constraints. 
We treat the soft SUSY breaking parameters $\left(m_{Q, U}^{2}, A_{u}\right), \mu$ and $\tan \beta$ as phenomenological inputs, assuming that the soft terms add in quadrature (see Eq. (12) 2. The radiative contribution to the (zero mode) CP-even Higgs mass-square matrix from the $n$th KK quark/squark mode is given by,

$$
\left.\Delta \mathcal{M}^{2}\right|_{(n) i j}=\left(N_{c} / 4 \pi^{2} v^{2}\right)\left(\Delta_{i j}^{u}\right)^{n},
$$

where, putting $\lambda^{(n)}=1$ following Eq. (9), and denoting $g(a, b) \equiv 2-\{(a+b) /(a-b)\} \ln (a / b)$,

$$
\begin{aligned}
\left(\Delta_{11}^{u}\right)^{n} & =\frac{v_{u}^{4}}{4 \sin ^{2} \beta}\left(\frac{\mu\left(A_{u}+\mu \cot \beta\right)}{m_{\tilde{u}_{1}^{n}}^{2}-m_{\tilde{u}_{2}^{n}}^{2}}\right)^{2} g\left(m_{\tilde{u}_{1}^{n}}^{2}, m_{\tilde{u}_{2}^{n}}^{2}\right), \\
\left(\Delta_{12}^{u}\right)^{n} & =\frac{v_{u}^{4}}{4 \sin ^{2} \beta} \frac{\mu\left(A_{u}+\mu \cot \beta\right)}{m_{\tilde{u}_{1}^{n}}^{2}-m_{\tilde{u}_{2}^{n}}^{2}}\left[\ln \frac{m_{\tilde{u}_{1}^{n}}^{2}}{m_{\tilde{u}_{2}^{n}}^{2}}+\frac{A_{u}\left(A_{u}+\mu \cot \beta\right)}{m_{\tilde{u}_{1}^{n}}^{2}-m_{\tilde{u}_{2}^{n}}^{2}} g\left(m_{\tilde{u}_{1}^{n}}^{2}, m_{\tilde{u}_{2}^{n}}^{2}\right)\right], \\
\left(\Delta_{22}^{u}\right)^{n} & =\frac{v_{u}^{4}}{4 \sin ^{2} \beta}\left[\ln \frac{m_{\tilde{u}_{1}^{n}}^{2} m_{\tilde{u}_{2}^{n}}^{2}}{m_{u^{n}}^{4}}+\frac{2 A_{u}\left(A_{u}+\mu \cot \beta\right)}{m_{\tilde{u}_{1}^{n}}^{2}-m_{\tilde{u}_{2}^{n}}^{2}} \ln \frac{m_{\tilde{u}_{1}^{n}}^{2}}{m_{\tilde{u}_{2}^{n}}^{2}}+\left(\frac{A_{u}\left(A_{u}+\mu \cot \beta\right)}{m_{\tilde{u}_{1}^{n}}^{2}-m_{\tilde{u}_{2}^{n}}^{2}}\right)^{2} g\left(m_{\tilde{u}_{1}^{n}}^{2}, m_{\tilde{u}_{2}^{n}}^{2}\right)\right] .
\end{aligned}
$$

The expressions for $\left(\Delta_{11}^{d}\right)^{n}$ can be written mutatis mutandis.

A comparison with what happens in flat space supersymmetric Universal Extra Dimension (UED) [13] is now in order. In UED, the KK states are equispaced (due to space-time flatness), and the KK Yukawa couplings are proportional to the corresponding zero mode masses. In the warped scenario, the KK states have a sparse spectrum following the zeros of the Bessel function, and the KK Yukawa couplings are, to a good approximation, independent of the flavor indices and are all close to unity for a reasonable choice of extra-dimensional parameters. In our warped SUSY scenario, for low and moderate $\tan \beta(\sim 10)$, only $m_{t}^{(1)} \sim 500 \mathrm{GeV}$ is light, which is a consequence of appropriately choosing the $c_{i}$ parameters for correctly reproducing zero mode fermion masses. For large $\tan \beta(\sim 40)$, although $m_{b}^{(1)}$ can be as light as $500 \mathrm{GeV}$, the prefactor $v_{d}^{4}$ that appears in $\left(\Delta_{i j}^{d}\right)^{n}$ (not shown explicitly) suppresses the radiative contribution to the Higgs mass induced by the down-type chiral multiplets. So in the warped case, only $u^{(1)}, c^{(1)}$ and especially $t^{(1)}$ multiplets contribute to $\Delta m_{h}^{2}$ in a numerically significant way. The contributions from higher KK states are negligible. This is in sharp contrast with the SUSY UED scenario where the first few $t^{(n)}$ (and not $u^{(n)}$ or $c^{(n)}$ ) chiral multiplets provide sizable contribution to $\Delta m_{h}^{2}$. The net numerical effects in the two cases are comparable. Recall that in UED, unlike in the warped case, the KK spectra are not linked to fermion mass hierarchy.

Numerical Results: For simplicity, we have assumed a common soft mass $M_{S} \equiv m_{Q}=m_{U}=m_{D}$. The trilinear couplings $A_{u}$ and $A_{d}$ have been varied in the range $[0.8-1.2] M_{S}$, which resulted in bands in the figures. In Fig. 1, we have plotted our results in the $m_{h}-m_{A}$ plane. We have used $m_{t}^{(1)}$, the lightest KK top quark mass (modulo the zero mode mass), to represent the extra-dimensional effect. In Fig. 1, we have displayed the effects for two choices of $m_{t}^{(1)}$, namely, 500 and $1000 \mathrm{GeV}$, and for a moderate $\tan \beta=10$. In Fig. 2, we have demonstrated that $m_{h}$ indeed falls with increasing $m_{t}^{(1)}$, eventually attaining its $4 \mathrm{~d}$ value. In this plot, we have set $A_{u}=A_{d}=\sqrt{6} M_{S}$, which maximises not only the 4d MSSM radiative correction but also the KK-induced one, which is why we have used the symbol $m_{h}^{\max }$. The three lines correspond to three different choices of $M_{S}=$ 500, 750 and $1000 \mathrm{GeV}$. All in all, $m_{h}$ increases by a few to several tens of a GeV, depending on the choice of soft SUSY breaking parameters, the radiative contribution coming primarily from all up-type multiplets. Beyond $m_{t}^{(1)}=2 \mathrm{TeV}$ (which corresponds to an average value of $\sim 6 \mathrm{TeV}$ for other first level KK fermions and gauge bosons), the KK effects almost vanish and the three falling curves become flat lines approaching their $4 \mathrm{~d}$ limits (of course, without ever meeting as the three $M_{S}$ values are different).

Conclusions: We have calculated one-loop correction to the lightest neutral Higgs boson mass in a generic MSSM embeded in a slice of $\mathrm{AdS}_{5}$. For a reasonable choice of warped space parameters, the 4d upper limit of $135 \mathrm{GeV}$ could be relaxed by as much as $\sim(50-100) \mathrm{GeV}$ depending on $M_{S}$. A few other closely related

\footnotetext{
${ }^{2}$ SUSY breaking by twisted boundary conditions on fermions leads to a linear splitting $\left(0.5 \pi k e^{-\pi k R}\right)$ ]. The numerical effects on $\Delta m_{h}^{2}$ would be covered within the parameter space we scanned (see figures later).
} 


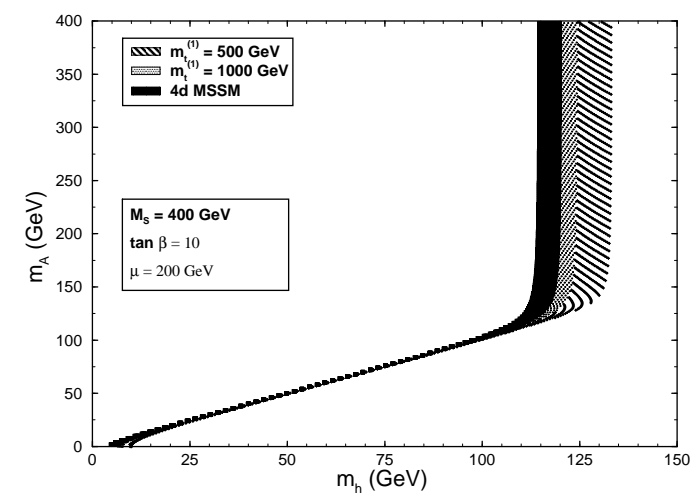

Figure 1: A comparison between 4d MSSM and 5d warped MSSM in the $m_{h}-M_{A}$ plane. The band width corresponds to the variation of $A_{u}$ and $A_{d}$ in the range $(0.8-1.2) M_{S}$.

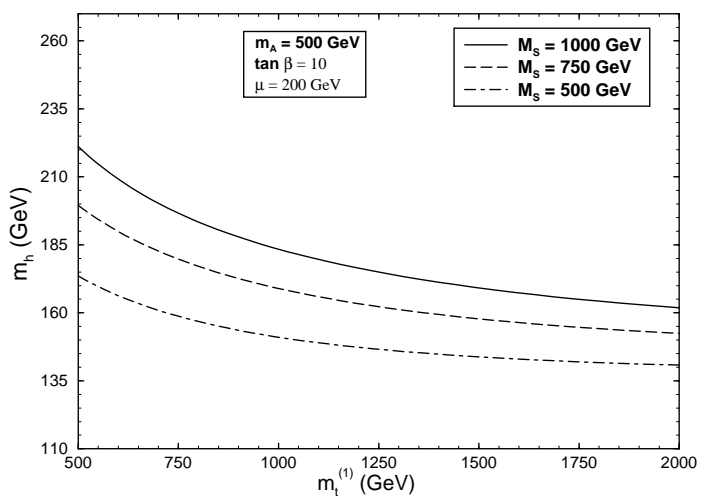

Figure 2: The variation of $m_{h}^{\max }$ with $m_{t}^{(1)}$ for different choices of $M_{S}$. We have used $A_{u}=A_{d}=\sqrt{6} M_{S}$ to maximise the radiative effect.

highlights are the following: (i) matter KK spectra are controlled by the $c_{i}$ parameters, which, in turn, are determined by the zero mode fermion masses; (ii) all KK Yukawa couplings are close to unity to a very good approximation; (iii) only $m_{t}^{(1)}$ could be light $(\sim 500 \mathrm{GeV})$ for a moderate $\tan \beta\left(m_{b}^{(1)}\right.$ can also be light too for large $\tan \beta \sim 40$ ) - this feature can be used to discriminate this scenario from the others producing similar shift to $m_{h}$; (iv) small values of $\tan \beta(\$ 3)$, which are otherwise disfavored in $4 \mathrm{~d}$ MSSM due to nonobservation of Higgs up to $114.5 \mathrm{GeV}$ [14, are now resurrected thanks to an additional KK-induced radiative corrections. Admittedly, the stability of the proton would require further care 3 . Besides, the warped models with fermions in the bulk, in general, pass the electroweak precision tests (EWPT) with some difficulty [15, unless the KK mass is raised to tens of a $\mathrm{TeV}$. To suppress excessive contribution to $T$ ( or $\Delta \rho$ ), gauge symmetry in the bulk is enhanced to $\mathrm{SU}(2)_{\mathrm{L}} \times \mathrm{SU}(2)_{\mathrm{R}} \times \mathrm{U}(1)_{\mathrm{B}-\mathrm{L}}$ [16], while to keep the contributions to $Z b_{L} \bar{b}_{L}$ vertex and other loop corrections under control, a further discrete $L \leftrightarrow R$ symmetry has been employed [17. This allows us to consider $m_{t}^{(1)}$ as light as $500 \mathrm{GeV}$ (the lightest KK gauge boson is then $1.5 \mathrm{TeV}$ ). Furthermore, $\tan \beta$ can be tuned to reduce the contribution to $T$. Since our primary intention here has been to develop a simple analytic framework to (for the first time) compute KK-induced radiative corrections to $m_{h}$ in a supersymmetric warped space, we pared the scenario down to its bare minimum. The further details necessary to overcome the above constraints are unlikely to alter the essential qualitative and quantitative features we explored here.

Finally, we note that in a general class of such models, KK-parity violating gauge interactions would induce the lightest KK particle decay into zero mode SM particles well before nucleosynthesis sets in, thus without disturbing any cosmological constraints. The dark matter candidate (which could very well be the lightest zero mode neutralino, provided $R$-parity is conserved) would still be decided on the basis of the $4 \mathrm{~d}$ spectra controlled by our choice of soft masses. Moreover, the lowest KK excitations of the SM particles could be heavier or lighter than the zero mode superparticles, again depending on our choice of the zero mode supersymmetry breaking soft parameters. A detailed phenomenological study preparing a catalogue of all such possibilities is beyond the goal of this paper.

Acknowledgements: We thank E. Dudas and A. Raychaudhuri for valuable comments. GB acknowledges hospitality at LPT-UMR 8627, Orsay, and a CNRS fellowship during a part of this work, and a partial support through the project No. 2007/37/9/BRNS of BRNS (DAE), India. TSR acknowledges the S.P. Mukherjee fellowship of CSIR, India.

\section{References}

[1] L. Randall and R. Sundrum, Phys. Rev. Lett. 83 (1999) 3370. 
[2] W. D. Goldberger and M. B. Wise, Phys. Rev. Lett. 83 (1999) 4922; W. D. Goldberger and M. B. Wise, Phys. Lett. B 475 (2000) 275; H. Davoudiasl, J. L. Hewett and T. G. Rizzo, Phys. Lett. B 473 (2000) 43; A. Pomarol, Phys. Lett. B 486 (2000) 153; S. Chang, J. Hisano, H. Nakano, N. Okada and M. Yamaguchi, Phys. Rev. D 62 (2000) 084025.

[3] T. Gherghetta and A. Pomarol, Nucl. Phys. B 586 (2000) 141.

[4] S. J. Huber and Q. Shafi, Phys. Lett. B 498 (2001) 256.

[5] Y. Grossman and M. Neubert, Phys. Lett. B 474 (2000) 361.

[6] K. R. Dienes, E. Dudas and T. Gherghetta, Nucl. Phys. B 567 (2000) 111.

[7] T. Gherghetta and A. Pomarol, Nucl. Phys. B 602 (2001) 3.

[8] K. Agashe, A. Belyaev, T. Krupovnickas, G. Perez and J. Virzi, Phys. Rev. D 77 (2008) 015003; K. Agashe, G. Perez and A. Soni, Phys. Rev. D 75 (2007) 015002.

[9] M. Drees, Int. J. Mod. Phys. A 4 (1989) 3635; U. Ellwanger and C. Hugonie, Phys. Lett. B 623 (2005) 93.

[10] Y. Zhang, H. An, X. d. Ji and R. N. Mohapatra, arXiv:0804.0268 [hep-ph].

[11] K. Agashe, A. Falkowski, I. Low and G. Servant, JHEP 0804 (2008) 027.

[12] See, for example, A. Djouadi, Phys. Rept. 459 (2008) 1, and references therein.

[13] G. Bhattacharyya, S. K. Majee and A. Raychaudhuri, Nucl. Phys. B 793 (2008) 114. See also, N. Uekusa, arXiv:0806.3229 [hep-ph].

[14] J. Alcaraz et al. [LEP Collaboration], "A combination of preliminary electroweak measurements and constraints on the standard model," arXiv:hep-ex/0612034.

[15] J. L. Hewett, F. J. Petriello and T. G. Rizzo, JHEP 0209 (2002) 030; C. Csaki, J. Erlich and J. Terning, Phys. Rev. D 66 (2002) 064021.

[16] K. Agashe, A. Delgado, M. J. May and R. Sundrum, JHEP 0308 (2003) 050.

[17] M. S. Carena, E. Ponton, J. Santiago and C. E. M. Wagner, Phys. Rev. D 76 (2007) 035006; M. S. Carena, E. Ponton, J. Santiago and C. E. M. Wagner, Nucl. Phys. B 759 (2006) 202. 